AGRICULTURE AND BIOLOGY JOURNAL OF NORTH AMERICA

ISSN Print: 2151-7517, ISSN Online: 2151-7525, doi:10.5251/abjna.2012.3.11.439.451

(C) 2012, ScienceHuß, http://www.scihub.org/ABJNA

\title{
Export trend of Indian ornamental fish industry
}

\author{
Liya Jayalal ${ }^{*}$, A. Ramachandran \\ School of Industrial Fisheries, Cochin University of Science and Technology, \\ Cochin-682 016, India. \\ ‘liyasanju@yahoo.com,rahul_rama@hotmail.com
}

\begin{abstract}
Inspite of having two hotspots of biodiversity India is way long back in the ornamental fish trade. Large number of species can only foster the needs of the industry. The study aims to (1) to find the various indigenous, exotic ornamental fish species and ornamental shrimp species being exported from India, (2) to provide an overview of the trends in the Indian ornamental fish export industry. 287 indigenous fish species, 92 exotic fish species and 44 ornamental shrimps have been found to get exported from India. The export trend of the industry for the past ten years shows a declining state which is also reflected in the annual and compound annual growth rate. Ornamental fish industry has enormous potential in tropical countries like India. To expand trade, new technologies and policies will have to be developed which will help in attaining a sustainable industry.
\end{abstract}

Keywords: India, Export, Annual Growth Rate, Compound Growth Rate

\section{INTRODUCTION}

Aquarium fish keeping as a hobby has a long history dating back to many centuries. Introduction of civil aviation after the Second World War expanded the hobby to a global industry (Tissera, 2010). Though the global ornamental fish trade is relatively small, it has a significant contribution to the trade in freshwater and marine aquatic products. The global ornamental fish trade in retail is worth more than US $\$ 8$ billion (MPEDA, 2010) while the exports was around US \$337 million (FAO, 2008). According to Rana (2007) Singapore continues to be the biggest exporter and also remains the Asian hub for ornamental fish while, US, EU and Japan are the major importers with Czech Republic having a prominent place in EU in ornamental fish trade.

Inspite of having two hotspots of biodiversity, India is way long back in the ornamental fish trade with an export worth US\$ 1.17 million during 2009-2010 (MPEDA, 2010). Out of 274 freshwater fish species from north eastern states only $32 \%$ of native fish are exported (Mahapatra et. al, 2007) and among 287 freshwater species from western ghats (Shaji et al., 2000), only 114 species are exported(Anon, 2005).

In ornamental fish trade the need is for large number of species or varieties in large quantities (Sane, 2007). According to Kawada (2007) non-uniqueness and limited specimens discouraged the Japanese importers to have trade with India initially. But the condition has changed with the introduction of Tetraodon travancoricus, Scarlet badis and Drape fin barbs as reported by the same author. The study aims to list the various indigenous and exotic ornamental fish species and ornamental shrimps getting exported from India and also to provide an overview of the trends in the Indian ornamental fish export industry. Though some workers have already listed the ornamental fish species exported from India, it mainly concentrates on the indigenous ornamental fish species.

\section{METHODOLOGY}

The study was carried out between 2009 and 2011 . The method for data collection consisted of information gathering from various ornamental fish exporters in India using an e-mail survey (Malhotra, 2001). The survey was conducted every two months to get the details of the fish species getting exported. From about 59 registered exporters only 26 were ready to co-operate with the survey. Besides the survey, various documents such as databases, reports, manuscripts and articles were also used to collect information. Secondary data was also collected from Marine Products Export Development Authority (MPEDA), Kochi, India and also from Ministry of Commerce, Government of India. The export data obtained from Ministry of Commerce, Government of India, were used to compute the 
annual growth and compound annual growth (Siegel, J.G., 1997). Annual growth and compound annual growth were computed to assess the growth of the ornamental export industry.

\section{ANNUAL GROWTH RATE $=$ (THIS YEAR-LAST YEAR)/LAST YEAR}

COMPOUND ANNUAL GROWTH RATE $=(($ LAST YEAR/FIRST YEAR) ^ $(1 / n-1))-1$

Where,

$\mathrm{n}=$ number of years

\begin{tabular}{|c|c|}
\hline FAMILY & NATIVE SPECIES (FRESHWATER) \\
\hline Adrianichthyidae & Oryzias dancena* \\
\hline \multirow{7}{*}{ Ambassidae } & Chanda nama* \\
\hline & Chanda ranga \\
\hline & Chanda thomassi* $^{*}$ \\
\hline & Chanda wolffii \\
\hline & Parambassis lala* \\
\hline & Parambassis ranga* \\
\hline & Parambassis thomassi(Day, 1870) \\
\hline Amblycipitidae & Amblyceps mangois \\
\hline \multirow{2}{*}{ Anabantidae } & Anabas cobojius* $^{*}$ \\
\hline & Anabas testudineus \\
\hline Anguillidae & Anguilla bengalensis \\
\hline \multirow{4}{*}{ Aplocheilidae } & Aplocheilus blockii \\
\hline & Aplocheilus lineatus(Valenciennes, 1846) \\
\hline & Aplocheilus panchax \\
\hline & Aplocheilus parvus* \\
\hline \multirow{5}{*}{ Badidae } & Badis badis \\
\hline & Badis badis assamensis \\
\hline & Badis blosyrus* \\
\hline & Badis kanabos* \\
\hline & Dario dario \\
\hline \multirow{16}{*}{ Bagridae } & Batasio batasio \\
\hline & Batasio fasciolatus* \\
\hline & Hemibagrus menoda* \\
\hline & Hemibagrus punctatus* \\
\hline & Horabagrus brachysoma \\
\hline & Horabagrus nigricollaris \\
\hline & Mystus bleekeri* \\
\hline & Mystus carico* \\
\hline & Mystus gulio (Hamilton, 1822) \\
\hline & Mystus tengara (Hamilton, 1822) \\
\hline & Mystus vittatus \\
\hline & Rama chandramara \\
\hline & Rita gogra \\
\hline & Rita rita(Hamilton, 1822) \\
\hline & Sperata aor (Hamilton, 1822) \\
\hline & Sperata seenghala \\
\hline \multirow{2}{*}{ Balitoridae } & Aborichthys elongates* \\
\hline & Aborichthys tikaderi* \\
\hline
\end{tabular}

\section{RESULTS AND DISCUSSION}

287 native fish species, 92 exotic fish species and 45 ornamental shrimps were found to be exported from India as ornamental fish. Among the 287 native fish species, 239 were freshwater fish and 48 were marine fish species. Among the indigenous freshwater species the largest number of species belongs to the family Cyprinidae. 98 species were found to belong to Cyprinidae. 16 species were found to belong to families Bagridae and Balitoridae. 12 species were found to belong to the families Channidae, Cobitidae and Sisoridae.

\section{Table1: Native Freshwater Fish Species found to be exported}




\begin{tabular}{|c|c|}
\hline & Acanthocobitis botia(Hamilton, 1822) \\
\hline & Acanthocobitis rubidipinnis \\
\hline & Mesonemacheilus guentheri ${ }^{\star}$ \\
\hline & Mesonoemacheilus triangularis(Day, 1865) \\
\hline & Nemacheilichthys rupelli \\
\hline & Schistura barapaniensis \\
\hline & Schistura beavani \\
\hline & Schistura corica \\
\hline & Schistura denisonii dayi \\
\hline & Schistura rupecola* \\
\hline & Schistura savona* \\
\hline & Schistura scaturigina \\
\hline & Schistura tigrinum* \\
\hline & Schistura vinciguerrae* $^{*}$ \\
\hline Belonidae & Xenentodon cancila \\
\hline Chacidae & Chaca chaca (Hamilton, 1822) \\
\hline & Channa amphibious \\
\hline & Channa aurantimaculata* \\
\hline & Channa barca \\
\hline & Channa bleheri \\
\hline & Channa diplogramma* \\
\hline & Channa gachua \\
\hline channidae & Channa Harcourt-Butleri \\
\hline & Channa marulius issabella (Rao) 1997 \\
\hline & Channa punctata \\
\hline & Channa special rainbow \\
\hline & Channa stewarti \\
\hline & Channa striata \\
\hline Chaudhuriidae & Pillaia indica* \\
\hline & Etroplus canarensis \\
\hline Cichlidae & Etroplus maculatus \\
\hline & Etroplus suratensis \\
\hline & Botia almorhae* $^{*}$ \\
\hline & Botia Dario \\
\hline & Botia lohachata \\
\hline & Botia rostrata \\
\hline & Botia striata (Gunther, 1868) \\
\hline & Lepidocephalichthys gunthea (Hamilton, 1822) \\
\hline cobitialae & Lepidocephalichthys menoni* $^{\star}$ \\
\hline & Lepidocephalus annandalei(Chaudhuri 1912) \\
\hline & Lepidocephalus goalparensis \\
\hline & Lepidocephalus thermalis (Valenciennes, 1846) \\
\hline & Pangio pangia \\
\hline & Somileptes gongota(Hamilton, 1822) \\
\hline & Barilius bakeri \\
\hline & Barilius barila* $^{*}$ \\
\hline & Barilius barna \\
\hline & Barilius bendalensis* \\
\hline & Barilius canarensis \\
\hline & Barilius dogarsinghi* $^{\star}$ \\
\hline Cyprinidae & Barilius evezardi* $^{*}$ \\
\hline & Barilius radiolatus * \\
\hline & Barilius shacra \\
\hline & Barilius tileo \\
\hline & Barilius vagra \\
\hline & Catla catla* \\
\hline & Chagunius chagunio* \\
\hline
\end{tabular}




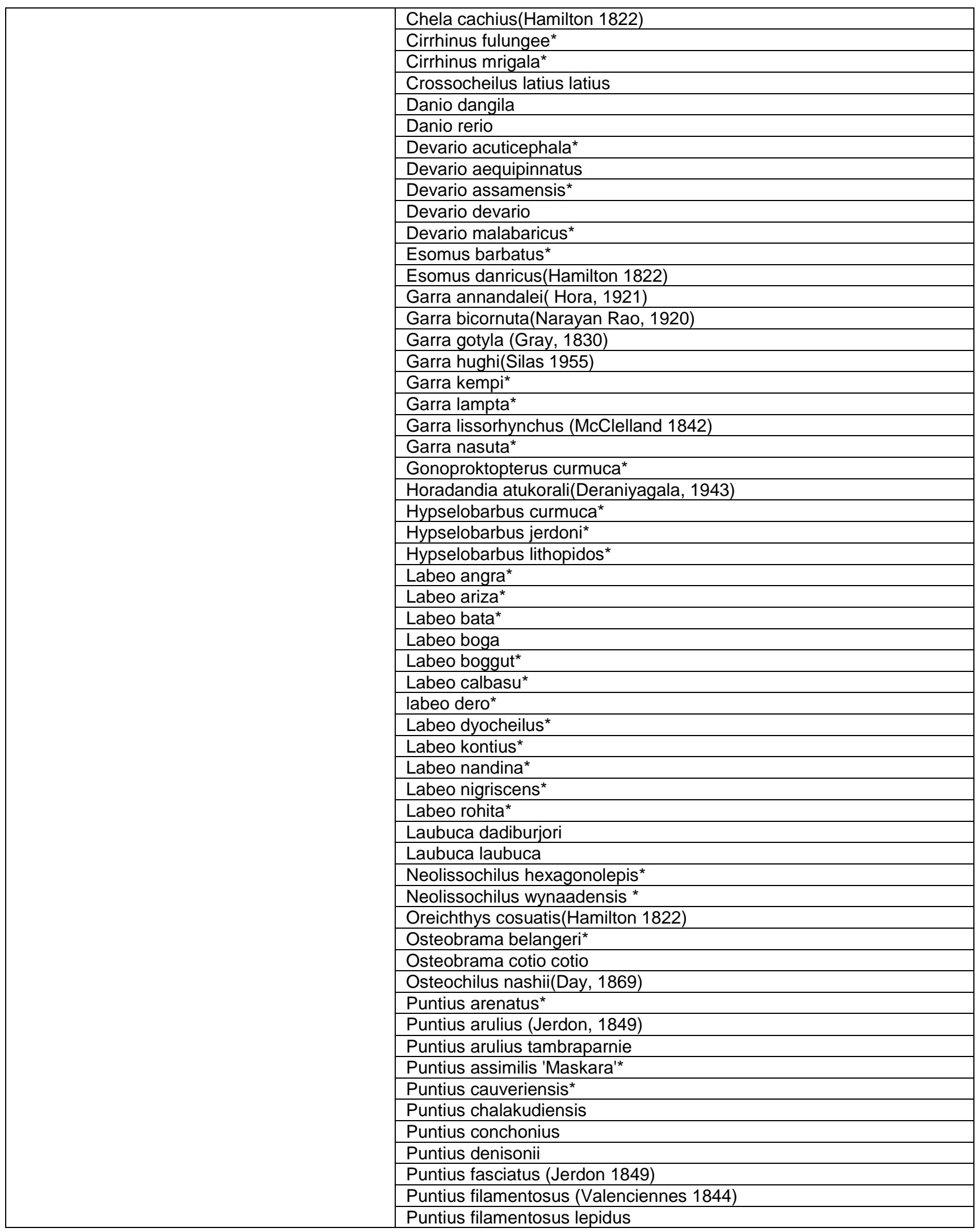




\begin{tabular}{|c|c|}
\hline & Puntius gelius \\
\hline & Puntius guganio(Hamilton, 1822) \\
\hline & Puntius jerdoni \\
\hline & Puntius mahecola(Valenciennes, 1844) \\
\hline & Puntius manipurensis* \\
\hline & Puntius meingangbii* \\
\hline & Puntius melanampyx \\
\hline & Puntius mudumalaiensis* \\
\hline & Puntius narayani(Hora, 1937) \\
\hline & Puntius phutunio(Hamilton 1822) \\
\hline & Puntius sahyadriensis(Silas, 1953) \\
\hline & Puntius setnai* \\
\hline & Puntius shalynius (Yazdani \& Talukdar 1975 \\
\hline & Puntius sophore (Hamilton 1822) \\
\hline & Puntius tambraparniei $^{*}$ \\
\hline & Puntius terio (Hamilton 1822) \\
\hline & Puntius ticto(Hamilton 1822) \\
\hline & Puntius vittatus(Day 1865) \\
\hline & Rajamas bola* \\
\hline & Rasbora daniconius (Hamilton, 1822) \\
\hline & Rasbora rasbora(Hamilton, 1822) \\
\hline & Rohtee ogilbi $^{*}$ \\
\hline & Salmophasia phulo* \\
\hline & Tor khudree \\
\hline & Tor mosal ${ }^{*}$ \\
\hline & Tor musullah \\
\hline & Tor progeneius* \\
\hline & Tor putitora* \\
\hline & Tor tor* \\
\hline & Erethistes pusillus * \\
\hline & Erethistoides montana* \\
\hline & Hara hara \\
\hline & Hara horai(Misra, 1976) \\
\hline Erethistidae & Hara jerdoni \\
\hline & Laguvia kapoori \\
\hline & Laguvia ribeiroi \\
\hline & Laguvia shawi \\
\hline & Pseudolaguvia muricata* \\
\hline & Brachygobius nunus \\
\hline & Glossogobius giurus (Hamilton 1822) \\
\hline Gobiidae & Gobiopterus chuno \\
\hline & Pseudapocryptes elongatus \\
\hline & Stigmatogobius sadanundio \\
\hline Heteropneustidae & Heteropneustes fossilis \\
\hline & Macrognathus aral \\
\hline Mastacembelidae & Macrognathus pancalus* \\
\hline & Mastacembalus armatus \\
\hline Moringuidae & Moringua raitaborua \\
\hline Mugilidae & Rhinomugil corsula \\
\hline & Nandus andrewi* \\
\hline & Nandus nandus \\
\hline Nandidae & Pristolepis malabaricus \\
\hline & Pristolepis marginata(Jerdon, 1849) \\
\hline & Chitala chitala* \\
\hline Notopteridae & Notopterus notopterus \\
\hline Olyridae & Olyra longicaudata( McClelland, 1842) \\
\hline
\end{tabular}




\begin{tabular}{|c|c|}
\hline \multirow{6}{*}{ Osphronemidae } & Ctenops nobilis( McClelland, 1845) \\
\hline & Polyacanthus fasciatus* \\
\hline & Polyacanthus lalius* \\
\hline & Polyacanthus sota* \\
\hline & Pseudosphromenus cupanus \\
\hline & Pseudosphromenus dayi* \\
\hline Pangasiidae & Pangasius pangasius(Hamilton, 1822) \\
\hline Schilbeidae & Neotropius atherinoides* \\
\hline \multirow{3}{*}{ Siluridae } & Ompok bimaculatus(Bloch, 1794) \\
\hline & Ompok pabda (Hamilton, 1822) \\
\hline & Wallago attu(Bloch \& Schneider, 1801) \\
\hline \multirow{12}{*}{ Sisoridae } & Bagarius bagarius(Hamilton, 1822) \\
\hline & Bagarius yelleri \\
\hline & Exostoma labiatum* \\
\hline & Gagata cenia \\
\hline & Gagata gagata* $^{*}$ \\
\hline & Gagata sexualis* \\
\hline & Glyptothorax cavia(Hamilton 1822) \\
\hline & Glyptothorax housei* $^{*}$ \\
\hline & Glyptothorax lonah* \\
\hline & Glyptothorax telchitta * \\
\hline & Gogangra viridescens \\
\hline & Sisor rhabdophorus(Hamilton, 1822) \\
\hline \multirow{2}{*}{ Synbranchidae } & Monopterus albus ${ }^{*}$ \\
\hline & Monopterus cuchia* \\
\hline Syngnathidae & Microphis deocata* \\
\hline \multirow{4}{*}{ Tetraodontidae } & Carinotetraodon imitator $^{\star}$ \\
\hline & Carinotetraodon travancoricus \\
\hline & Tetraodon cutcutia \\
\hline & Tetraodon fluviatilis \\
\hline
\end{tabular}

* new species added after list by Sekharan(2006)

Among the native marine water fish, 13 species were found to belong to the family Gobiidae , 4 species in the family Muraenidae and 3 species in the family Ariidae.

Among the exotic ornamental fish that were exported from India, 19 species belonged to the family Cyprinidae, 9 species belonged to the family Cichlidae and 5 species belonged to the family Osphronemidae.
Ramachandran (2002) had listed 172 indigenous freshwater fish species as being exported and Sekharan (2006) had listed 319 indigenous freshwater fish species as being exported.Works of authors like Ponniah \& Gopalakrishnan (2000), Ramachandran (2002), Sekharan (2006), Remadevi and Indra ( 2009 ) and the website Fishbase were reviewed to classify the exported ornamental fish as indigenous and exotic. 
Table2: Native Marine Fish Species Found to be exported

\begin{tabular}{|c|c|}
\hline Family & NATIVE SPECIES (MARINE WATER) \\
\hline \multirow{3}{*}{ Ariidae } & Arius dussumieri (Valenciennes 1840)* \\
\hline & Arius sagor ${ }^{*}$ \\
\hline & Arius sona \\
\hline Batrachoididae & Allenbatrachus grunniens ${ }^{*}$ \\
\hline \multirow{2}{*}{ Blenniidae } & Istiblennius edentulus* \\
\hline & Omobranchus zebra* \\
\hline Carangidae & Gnathanodon speciosus* \\
\hline Chaetodontidae & Chaetodon collare* \\
\hline Cynoglossidae & Cynoglossum semifasciatus* \\
\hline \multirow{2}{*}{ Eleotridae } & Eleotris fusca \\
\hline & Ophiocara aporos* \\
\hline \multirow{14}{*}{ Gobiidae } & Acentrogobius viridipunctatus(Valenciennes, 1837$)^{*}$ \\
\hline & Apocryptes bato \\
\hline & Boleophthalmus boddarti \\
\hline & Drombus globbiceps* \\
\hline & Gobiopsis macrostoma \\
\hline & Odontamblyopus rubicundus (Hamilton, 1822) \\
\hline & Oligolepis acutipennis (Valenciennes, 1837) \\
\hline & Oxyurichthys microlepis* \\
\hline & Periophthalmus dipus* \\
\hline & Periophthalmus pearsei \\
\hline & Pseudapocryptes lanceolatus* \\
\hline & Scartelaos histophorus \\
\hline & Taenoides Cirratus* \\
\hline & Trypauchen vagina * \\
\hline Hemiramphidae & Hyporhamphus limbatus* \\
\hline Lactidae & Lates calcarifer \\
\hline Lutjanidae & Lutjanus argentimaculatus ${ }^{*}$ \\
\hline Monodactylidae & Monodactylus argenteus* \\
\hline \multirow{2}{*}{ Muraenesocidae } & Congresox talabon 'hi-fin conger eel'* \\
\hline & Congresox talabonoides* \\
\hline \multirow{3}{*}{ Muraenidae } & Gymnothorax sathete* \\
\hline & Gymnothorax tile* \\
\hline & Lycodontis tile \\
\hline
\end{tabular}




\begin{tabular}{|c|c|}
\hline & Siderea thyrsoidea* \\
\hline Ophichthidae & Pisodonophis boro \\
\hline Ostraciidae & Lactoria cornuta* \\
\hline \multirow{2}{*}{ Platycephalidae } & Platycephalus bengalensis* \\
\hline & Platycephalus indicus* \\
\hline Plotosidae & Plotosus canius \\
\hline Ptereleotridae & Parioglossus pillipinus* \\
\hline Scatophagidae & Scatophagus argus argus \\
\hline \multirow{2}{*}{ Sillaginidae } & Sillaginopsis panijus \\
\hline & Sillago sihama* \\
\hline Soleidae & Euryglossa pan* \\
\hline Sparidae & Acanthopagrus berda(Forsskal, 1775) ${ }^{*}$ \\
\hline Syngnathidae & Syngnathus spicifer ${ }^{*}$ \\
\hline Tetraodontidae & Chelondon patoca \\
\hline
\end{tabular}

Table2: Native Marine Fish Species Found to be exported

* new species added after list by Sekharan(2006)

Table 3: Exotic fish species

\begin{tabular}{|l|l|}
\hline Family & EXOTIC FISH SPECIES \\
\hline Acanthuridae & Zebrasoma veliferum \\
\hline Actiniidae & Anthopleura xanthogrammica \\
\hline Ampullaridae & Pomacea bridgesii \\
\hline Anostomidae & Anostomus ternetzi \\
\hline Ariidae & Hexanematichthys seemanni \\
\hline Atyidae & Typhlatya iliffei \\
\hline Auchenipteridae & Ageneiosus marmoratus \\
\hline Badidae & Badis burmanicus \\
\hline \multirow{2}{*}{ Balitoridae } & Schistura macrocephalus \\
\hline Blenniidae & Ecsenius axelrodi \\
\hline \multirow{3}{*}{ Callichthyidae } & Corydoras aeneus \\
\cline { 2 - 2 } & Corydoras julii \\
\cline { 2 - 2 } & Corydoras paleatus \\
\hline Centrarchidae & Lepomis gibbosus \\
\hline Channidae & Channa pulchra \\
\hline \multirow{3}{*}{ Characidae } & Gymnocorymbus ternetzi \\
\cline { 2 - 2 } & Hemigrammus anisitsi \\
\cline { 2 - 2 } & Hyphessobrycon serape \\
\hline Cichlidae & Astronotus ocellatus \\
\hline
\end{tabular}


Agric. Biol. J. N. Am., 2012, 3(11): 439-451

\begin{tabular}{|c|c|}
\hline & Cichlasoma severum \\
\hline & Hemichromis paynei \\
\hline & Herichthys cyanoguttatus \\
\hline & Julidochromis \\
\hline & Pseudotropheus greshakei \\
\hline & Pseudotropheus zebra \\
\hline & Pterophyllum scalare \\
\hline & Thorichthys meeki \\
\hline Claridae & Clarias batrachus \\
\hline & Clarias macrocephalus \\
\hline & Botia kubotai \\
\hline Cobitidae & Chromobotia macracanthus \\
\hline & Cobitis striata \\
\hline & Barbus apleurogramma \\
\hline & Barbus quadripunctatus \\
\hline & Brachydanio kerri \\
\hline & Carassius auratus \\
\hline & Crossocheilus siamensis \\
\hline & Danionella translucida \\
\hline & Epalzeorhychus frenatus \\
\hline & Epalzeorhynchus bicolor \\
\hline & Garra rufa \\
\hline Cyprinidae & Hypsibarbus wetmorei \\
\hline & Labeo chrysophekadion \\
\hline & Laubuca caeruleostigmata \\
\hline & Puntius lineatus \\
\hline & Puntius nigrofasciatus \\
\hline & Puntius orphoides \\
\hline & Puntius semifasciolatus \\
\hline & Puntius tetrazona \\
\hline & Rasbora trilineata \\
\hline & Tanichthys albonubes \\
\hline Doraridae & Platydoras costatus \\
\hline Eleotridae & Eleotris marmorata \\
\hline Erethistidae & Hara filamentosa \\
\hline Gnathophyllidae & Hymenocera picta \\
\hline Gobiidae & Brachygobius doriae \\
\hline & Gobiodon ceramensis \\
\hline
\end{tabular}


Agric. Biol. J. N. Am., 2012, 3(11): 439-451

\begin{tabular}{|c|c|}
\hline & Gobiosoma oceanops \\
\hline & Gobius tigrellus \\
\hline Haemulidae & Plectorhinchus vittatus \\
\hline Helostomatidae & Helostoma temminckii \\
\hline I ricariidao & Hypostomus plecostomus \\
\hline & Rineloricaria fallax \\
\hline Lutjanidae & Lutjanus campechanus \\
\hline Mastacembelidae & Macrognathus siamensis \\
\hline Muraenidae & Gymnothorax moringa \\
\hline Mysidae & Praunus flexuosus \\
\hline Nothobranchiidae & Epiplatys chevalieri \\
\hline Ocypodidae & Uca perplexa \\
\hline & Betta splendens \\
\hline & Trichogaster leeri \\
\hline Osphronemidae & Trichogaster microlepis \\
\hline & Trichogaster trichopterus \\
\hline & Trichopodus trichopterus \\
\hline Osteoglossidae & Scleropages jardinii \\
\hline Pangasiidae & Pangasius sutchi \\
\hline Parathelphusidae & Austrothelphusa transversa \\
\hline Pimelodidae & Leiarius pictus \\
\hline & Poecilia latipinna \\
\hline Poecilidae & Poecilia sphenops \\
\hline & Xiphophorus helleri \\
\hline Pomacanthidae & Centropyge aurantia \\
\hline Potamonidae & Parathelphusa martens \\
\hline Ptereleotridae & Parioglossus palustris \\
\hline Rhynchocinetidae & Rhynchocinetes rigens \\
\hline Scyliorhinidae & Apristurus gibbosus \\
\hline Sebastidae & Helicolenus percoides \\
\hline Stichodactylidae & Stichodactyla haddoni \\
\hline Stomidae & Stomias boa boa \\
\hline Syngnathidae & Indian pipefish/ichthyocampus carce \\
\hline dae & Takifugu niphobles \\
\hline & Tetraodon mbu \\
\hline
\end{tabular}

Table 3: Exotic fish species 
Table 4: Ornamental shrimps being exported

\begin{tabular}{|c|}
\hline SHRIMPS \\
\hline Caradina Sp./Zigzag Shrimp \\
\hline Caradina tiwari \\
\hline Caridina babaulti \\
\hline Caridina gracileps \\
\hline Caridina gracilirostris \\
\hline Caridina hodgarti \\
\hline Caridina new 'Black Beauty' \\
\hline Caridina Sp./Black banded Green Shrimp \\
\hline Caridina Sp./Black banded Shrimp \\
\hline Caridina Sp./Black Shrimp \\
\hline Caridina Sp./Dwarf Green Shrimp \\
\hline Caridina Sp./Fire Head shrimp \\
\hline Caridina Sp./Green Shrimp \\
\hline Caridina Sp./lvory Shrimp \\
\hline Caridina Sp./Zebra Shrimp \\
\hline Macrobrachium assamensis \\
\hline Macrobrachium banjare(Tiwari 1958) \\
\hline Macrobrachium duarii \\
\hline Macrobrachium dulichodctileus \\
\hline Macrobrachium idella \\
\hline Macrobrachium kulsiense \\
\hline Macrobrachium naso \\
\hline Macrobrachium peguensis \\
\hline Macrobrachium pilimanus \\
\hline Macrobrachium scabriculum(Heller, 1862) \\
\hline Macrobrachium sp. 'Himalayanus' \\
\hline Macrobrachium sp. 'Ivory' \\
\hline Macrobrachium Sp./Bicolour Shrimp \\
\hline Macrobrachium Sp./Black Leaf Shrimp \\
\hline Macrobrachium Sp./Green Leaf Shrimp \\
\hline Macrobrachium Sp./Pigeon Blood Shrimp \\
\hline Macrobrachium Sp./Red Claw shrimps \\
\hline Macrobrachium Sp./Redtail Fancy Shrimp \\
\hline Macrobrachium tiwari \\
\hline Palaemon emerald \\
\hline Palaemon hendersoni \\
\hline
\end{tabular}

Palaemon scabriculus

Palaemon scarletti

Palaemon sp. 'green needlenose'

Palaemon sp. 'Yellow fluorescent'

Palaemon Sp./Blue Band Shrimp

Palaemon Sp./Green Rocket Shrimp

Penaeus brasiliensis

Zico Shrimp

Recent trade statistics from Ministry of Commerce, Government of India, indicates that the export demand for ornamental fish is declining (Fig 1.). The annual growth rate and compound annual growth rate also indicates a decline in the exports (Table 5)

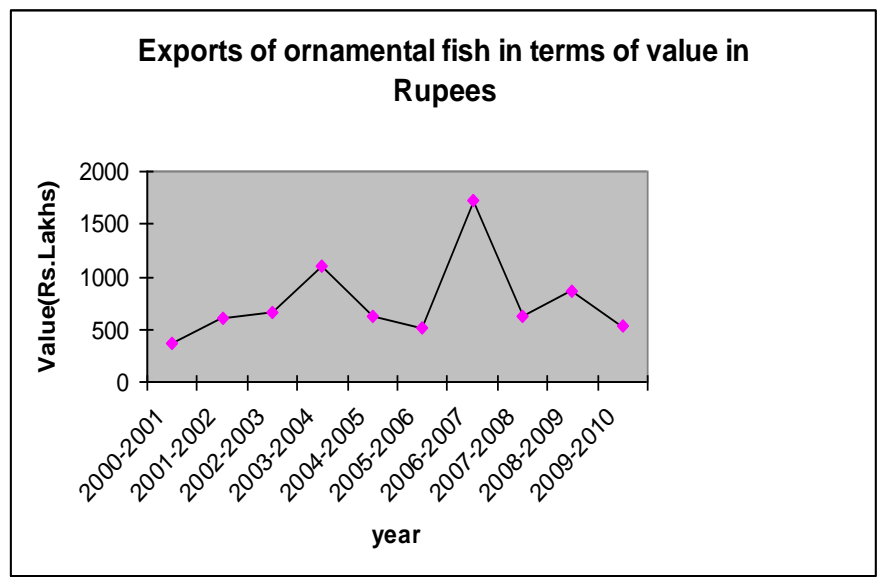

Fig 1: Exports of Ornamental fish in Terms of Value in Rupees (Lakhs)

Source: Compiled using data from the Ministry of Commerce, Gol.

Though the exports increased twice it substantially declined after 2007-2008. The reasons could be due to drastically reduced exports to Singapore, which is considered to be the main importer of Indian ornamental fish (Rana, 2007), from Rs.1079.22 lakhs in 2006-2007 to Rs. 252.42 lakhs (Table 6). Another reason could be the regulation imposed by Government of Kerala, regarding the catching of Puntius denisonii. According to fbas (2009), it is one of the most sought after ornamental fish from India and accounted for almost $60-65 \%$ of India's total live ornamental fish exports during the year 2007-2008. 
Table 5: Annual Growth Rate and Compound Annual Growth Rate of Ornamental fish Exports in Terms of Value in Rupees

\begin{tabular}{|l|l|l|}
\hline Year & Annual Growth (\%) & Compound Annual Growth Rate (\%) \\
\hline $2000-2001$ & & \\
\hline $2001-2002$ & 63.93 & 63.94 \\
\hline $2002-2003$ & 9.2 & 33.8 \\
\hline $2003-2004$ & 66.48 & 43.91 \\
\hline $2004-2005$ & -43.84 & 13.74 \\
\hline $2005-2006$ & -16.69 & 6.87 \\
\hline $2006-2007$ & 234.38 & 29.25 \\
\hline $2007-2008$ & -64.04 & 7.66 \\
\hline $2008-2009$ & 38.94 & 11.14 \\
\hline $2009-2010$ & -39.2 & 3.936 \\
\hline
\end{tabular}

Source: Computed by the author

Table 6: Annual Growth Rate and Compound Annual Growth Rate of Ornamental fish Exports in Terms of Value in Rupees

\begin{tabular}{|l|l|l|l|l|l|l|l|l|l|l|}
\hline Country & $\mathbf{2 0 0 0 -}$ & $\mathbf{2 0 0 1 -}$ & $\mathbf{2 0 0 2 -}$ & $\mathbf{2 0 0 3 -}$ & $\mathbf{2 0 0 4 -}$ & $\mathbf{2 0 0 5 -}$ & $\mathbf{2 0 0 6 -}$ & $\mathbf{2 0 0 7 -}$ & $\begin{array}{l}\mathbf{2 0 0 8 -} \\
\mathbf{2 0 0 0}\end{array}$ & $\begin{array}{l}\mathbf{2 0 0 9 -} \\
\mathbf{2 0 1 0}\end{array}$ \\
\hline France & 1.94 & 3.83 & 4.18 & 3.06 & 1.62 & 1.53 & 2.52 & 1.01 & 0.02 & 7.86 \\
\hline Germany & 27.31 & 16.45 & 26.31 & 28.09 & 37.01 & 30.36 & 27.99 & 34.8 & 41.67 & 54.02 \\
\hline Hongkong & 69.59 & 104.89 & 10.18 & 59.44 & 80.55 & 60.49 & 52.96 & 45.1 & 42.58 & 47.72 \\
\hline Japan & 100.29 & 58.25 & 198.17 & 406.47 & 56.82 & 48.08 & 52.59 & 40.46 & 67.21 & 46.69 \\
\hline Malaysia & 7.99 & 1.71 & 2.97 & 224.35 & 25.23 & 87.05 & 52.23 & 53.4 & 38.53 & 39.07 \\
\hline Netherlands & 8.92 & 4.26 & 7.48 & 7.49 & 8.51 & 12.36 & 18.05 & 15.49 & 12.35 & 14.58 \\
\hline Singapore & 60.01 & 50.99 & 66.89 & 82.14 & 140.53 & 134.9 & $1,079.22$ & 252.42 & 265.43 & 172.01 \\
\hline Srilanka & 0.66 & 11.54 & 4.4 & 5.56 & 2.28 & 3.62 & 2.18 & 4.13 & 0.33 & 0.56 \\
\hline Taiwan & 3.64 & 252.34 & 3.17 & 34.64 & 19.87 & 7.93 & 3.2 & 2.1 & 5.08 & 6.42 \\
\hline Thailand & 14.24 & 3.43 & 80.49 & 3.3 & 9.47 & 7.17 & 39.45 & 0.6 & 1.21 & 16.06 \\
\hline UAE & 1.34 & 6.09 & 20.08 & 40.26 & 14.1 & 3.55 & 205.05 & 40.5 & 232.69 & 1.43 \\
\hline UK & 11.33 & 12.58 & 73.83 & 21.93 & 24.08 & 10.65 & 21.56 & 33.49 & 13.44 & 15.27 \\
\hline USA & 53.87 & 69.79 & 72.62 & 93.93 & 66.73 & 58.45 & 44.59 & 37 & 46.11 & 56.96 \\
\hline
\end{tabular}

Table 6: Exports in terms of Value in Rupees (Lakhs)

Source: Computed using data from the Ministry of Commerce, Gol.

\section{CONCLUSION:}

Ornamental fish industries have enormous potential in tropical countries. Avenues are unlimited to India. For the trade to prosper three pre-requisites are quality, quantity and sustainability (MPEDA, 2010). Therefore, the exploitation of wild fish stocks for the aquarium trade may become restricted due to a trend towards conserving the country's natural resources. So to expand the trade, new technologies will need to be developed in order to commercially breed rare species, as well as marines. Proper fish health management and quarantine regimes will also have to be adopted. Besides these, to achieve an ornamental fish industry which is sustainable, policies should also take into care the interests and welfare of the stakeholders associated with the industry.

\section{REFERENCES}

Anon (2005) List of freshwater and brackish water ornamental fishes that are being exported from India, In: MPEDA Newsletter July 2005, pp 10-17.

FAO (2008) Accessed through www.fao.org/fi/statist/FISOFT/FISHPLUS.asp.

Kawada, I. (2007) Market of ornamental fish in Japan, Paper presented in technical session of INDAQUA 2007 11-13 January, Chennai. 
Mahapatra, B.K., Vinod, K., and Mandal B.K. (2007) Inland indigenous ornamental fish germplasm of northeastern India: status and future strategies, In: Biodiversity and its significance (Tandon, P., Abrol, Y.P., and Kumaria, S., Eds), pp 134-149.

Malhotra, N.K. (2001) Marketing research, $1^{\text {st }}$ edn., Addison Wesley Longman Pvt.Ltd., New Delhi, India, $761 \mathrm{p}$.

Mital, R. (2009) Conserving Miss Kerala In: Federation of British Aquatic Sciences Bulletin, Autumn 2009. Accessed http://www.fbas.co.uk/September\%20issue6A.pdf

Silas, E.G., Gopalakrishnan, A., Ramachandran, A., Anna Mercy, T.V., Kripan Sarkar, Pushpangathan, K.R., Anil Kumar, P., Ram Mohan, M.K., and Anikuttan, K.K. (2011) Guidelines for green certification of freshwater ornamental fish. The Marine Products Development Authority, Kochi.

Ponniah, A.G., and Gopalakrishnan, A. (2000) Cultivable, ornamental, sport and food fishes endemic to peninsular India with special refernce to Western Ghats, In: Endemic fish diversity of Western Ghats (Ponniah, A.G., and Gopalakrishnan, A., Eds) NBFGRNATP Publication, pp 13-32.

Ramachandran, A. (2002). Freshwater indigenous ornamental fish resources in Kerala and their prospects for international marketing, In: Riverine and reservoir fisheries of India (Boopendranath, M.R., Meenakumari, B., Joseph, J., Sankar, T.V., Pravin, P. and Edwin, L. Eds.) pp 109-134, Society of Fisheries Technologists (India), Cochin.
Rana, K.J. (2007). International ornamental fish trade: supply lines, markets and regulations in key marketsglobal trade in ornamental fish, Paper presented in technical session of Indaqua 2007 11-13 January, Chennai.

Remadevi, K., and Indra T.J. (2009) Checklist of the native freshwater fishes of India, Zoological Survey of India.

Sane, S. (2007) Understanding ornamental fish trade in India, Paper presented in technical session of Indaqua 2007 11-13 January, Chennai.

Sekharan, M. (2006) Prospects of marketing the indigenous ornamental fishes of Kerala. PhD Thesis. Cochin University of Science and Technology, Kochi, Kerala, India.

Shaji, P.C, Easa, P.S. and Gopalakrishnan, A. (2000) Freshwater fish diversity of western ghats, In: Endemic fish diversity of Western Ghats (Ponniah, A.G., and Gopalakrishnan, A., Eds) NBFGR-NATP Publication, pp 13-32.

Siegel, J.G., Shim, K.J. and Hartman, S. (1997) Schaum's quick guide to business formulas: 201 decision-making tools for business, finance, and accounting students, pp 70

Tissera, K. (2010) Global trade in ornamental fishes-1998 to 2007, Paper presented at International Aquashow 12-14 February, Cochin, Kerala, India. 